Review

\title{
Pathophysiologic Significance of Host Reactions in Human Cancer Tissue: Desmoplasia and Tumor Immunity
}

\author{
Haruo Ohtani \\ Department of Pathology, Tohoku University School of \\ Medicine, Sendai 980-8575
}

\begin{abstract}
Ohtani, H. Pathophysiologic Significance of Host Reactions in Human Cancer Tissue: Desmoplasia and Tumor Immunity. Tohoku J. Exp. Med., 1999, 187 (3), 193-202 — Invasive growth of malignant cells, particularly carcinoma cells, induces host reaction within and around tumor tissue. Representatives of them are desmoplasia, angiogenesis and immune reactions. Desmoplasia, a process of fibrosis, is induced by activation of fibroblasts with increased production of matrix proteins and matrix degrading enzymes. Angiogenesis is prerequisite for the growth of solid tumor. Inhibition of this is now a target of cancer therapy. The present author has proposed a concept that tumor vessels are composed of nutrient vessels and immune/inflammatory vessels. The latter is similar to venules in inflammatory lesions expressing the cell adhesion molecules to facilitate the transmigration of inflammatory cells to the tissue. In colon cancer, venules distributed along the invasive margin correspond to these vessels, which express E-, and P-selectins, and ICAM-1. These venules are considered to be an entry site of immune/inflammatory cells to cancer tissue. To further analyze immune mechanism, the present authors have confirmed that macrophages distributed along the invasive margin of colon cancer express costimulatory molecules B7.1/B7.2, which are required for the proliferation of T-cells. T-cells were co-localized with these cells. Clinicopathologic analysis confirmed that $\mathrm{CD}^{+} \mathrm{T}$-cells distributed within cancer cell nest (intraepithelial) have the most significant impact on the patients' survival in colorectal cancer. These data suggest that various host reactions take place in the stroma of cancer tissue, which modulate the biologic behavior of cancer. - stromal reactions; cancer invasion; immune reaction; desmoplasia (C) 1999 Tohoku University Medical Press
\end{abstract}

Growth of cancer cells, particularly invasive growth beyond its orthotopic location, induces various host reactions in the stroma. Host reactions are comprised of desmoplasia, angiogenesis, and immune reactions. These reactions have

Received and accepted for publication February 23, 1999.

Address for reprints: Haruo Ohtani, M.D., Department of Pathology, Tohoku University School of Medicine, 1-1 Seiryomachi, Aoba-ku, Sendai 980-8575, Japan.

e-mail: hohtani@patholo2.med.tohoku.ac.jp

Dr. H. Ohtani is a recipient 1998 of the Gold Prize, Tohoku University School of Medicine. 
been regarded to modulate the biologic behavior of cancer. Some of them are on the clinical trials including the inhibition of angiogenesis or the augmentation of immune reactions. However, not so much attention has been paid to these host reactions in cancer in the morphologic studies, leaving large fields unclarified. The present paper reviews studies by the present author on the possible pathophysiologic significance of host reactions in the stroma of the cancer based on the morphologic analysis. The author will show that studies on the stromal reaction are one of mandatory clues to analyze the biologic behavior of human cancers.

\section{Desmoplastic reactions (fibrosis)}

When cancer cells, especially epithelial malignant cells (carcinoma cells) start to invade beyond their orthotopic locations, the host frequently react with desmoplastic reactions (increased fibrosis) (Ohtani and Sasano 1983). Hepatocellular and renal carcinomas are representative exceptions, whose stroma is mainly composed of blood vessels usually devoid of desmoplasia. It has been generally conceived that collagen fibers are produced by fibroblasts. Based on in vitro experiments, another theory stressed the possibility that it was carcinoma cells that produce collagen (Sakakibara et al. 1982). To determine the origin of collagen fibers, exact analysis on human cancer tissue is prerequisite. Immunohistochemistry for collagen molecules mainly stains collagen fibers deposited in the extracellular matrix, making identification of cells that produce collagen difficult. The morphologic method to identify the cell type is two: Immunolectron microscopy to identify labeling the lumen of rough endoplasmic reticulum (demonstration of production of proteins with a signal peptide) or in situ hybridization to demonstrate cells expressing the corresponding mRNA. Immunoelectron microscopy for both types I and III collagen revealed a labeling in the lumen of rough endoplasmic reticulum in fibroblasts but not in cancer cells in gastrointestinal carcinoma. Furthermore, in situ hybridization for type I procollagen revealed positive signals in stromal fibroblasts, not in cancer cells (Ohtani et al. 1992). These results are also the same in diffuse-type gastric cancer, which is characterized by remarkable deposition of collagen fibers in the stroma. Therefore, we concluded that cells responsible for the production of collagen in cancer tissue are mainly stromal fibroblasts. In the present study the author uses the term fibroblasts in a wider sense including myofibroblasts, since most of stromal fibroblasts in cancer tissue have bundles of actin-filaments (Ohtani and Sasano 1983).

To clarify the involvement of growth factors/cytokines in this mechanism, we studied the tissue expression of TGF- $\beta$ and latent TGF- $\beta$ binding protein (LTBP) (Mizoi et al. 1993). The immunohistochemical expression of TGF- $\beta$ is more apparent in diffuse-type gastric carcinoma. Positive cells included both cancer cells and fibroblasts. Immunolectron microscopy revealed that carcinoma cells were labeled diffusely in the cytoplasm (cytoplasmic matrix) while fibroblasts 
showed labeling in the lumen of rough endoplasmic reticulum. The labeling pattern of fibroblasts is typical for a secretory-type protein, but not in cancer cells. LTBP is solely positive in fibroblasts. These observations suggested that fibroblast could secrete $\mathrm{TGF}-\beta$ more efficiently than cancer cells in gastric cancer tissue. More abundant expression of TGF- $\beta$ in diffuse-type carcinoma may be related to a deposition of mature collagen fibers in this type. After the submission of our paper, the concept of immunosuppression by TGF- $\beta$ was proposed (Shull et al. 1992), which signified that TGF- $\beta$ is also one of immunosuppressive growth factors in cancer tissue.

The pathophysiologic significance of desmoplastic reaction remains unclear. We have dealt with seprase, a member of serine integral membrane proteases. This transmembrane proteinase has a gelatinolytic activity and has been found on invadopodia of melanoma cells in vitro (Goldstein et al. 1997; Pineiro-Sanchez et al. 1997). This indicates its involvement in cell invasion mechanism. The monomer form of seprase is nearly identical to fibroblast activation protein alpha (Scanlan et al. 1994). We have performed immunohistochemical study of seprase in breast cancer revealing that fibroblasts were positive while cancer cells were negative. These results suggest that seprase is expressed as a fibroblastic reaction occurring in the stroma. Clinicopathologic analysis revealed that a more abundant seprase expression was associated with a better survival, particularly in patients with lymph node metastasis or with a higher tumor grade. This suggests that stromal fibroblasts possess a suppressive effect to cancer growth in breast cancer (Ariga et al. manuscript in preparation).

A new aspect of the concept of "similarity between tumor stroma generation and wound repair process"

One of the most intriguing hypotheses in this field is the similarity between tumor stroma generation and wound repair process (Dvorak 1986). The similarity includes the sequential process beginning from hyperpermeability of blood vessels, fibrin formation, fibrinolysis, fibrosis and ending up with scarring. Our above-mentioned studies also demonstrated the similarity of seprase expression between cancer stroma and wound repair process (granulation tissue reaction). We studied MMP-2, another important matrix degrading enzymes, which is widely expressed by stromal fibroblasts in various cancer tissue (Poulsom et al. 1992; Pyke et al. 1992; Heppner et al. 1996). We confirmed that the overexpression patterns of MMP-2 and type I procollagen is commonly observed in wound repair process and cancer stroma in the gastrointestinal tract (Ohtani et al. 1995). Based on these observations, the present author proposed that the similarity between the cancer stroma generation and wound repair process includes the production of certain matrix-degrading enzymes, which is probably related to the turnover of matrix proteins, i.e., tissue remodeling process (Ohtani 1998) (Fig. 1). Current studies on matrix degrading enzymes are done from the standpoint of cancer cell 


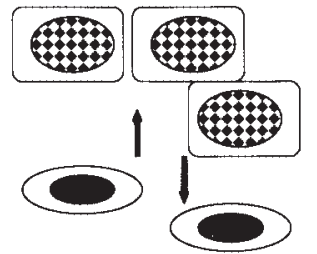

Cancer stroma

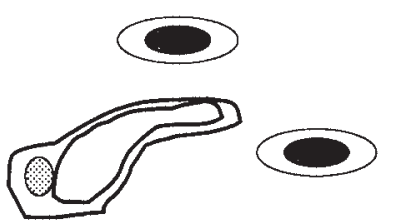

Wound repair (granulation tissue)

---- Common features ----

- Fibrin deposition, Hyperpermeability of blood vessels*

- Fibrinolysis*

- Fibrosis*

- Production of matrix-degrading enzymes ${ }^{\star \star}$

Seprase, MMP-2....

Fig. 1. Similarity between cancer stroma and wound repair process (revised from Dvorak 1986).

Fibroblasts are activated in cancer stroma to produce matrix proteins and matrix degrading enzymes, which are the same as in fibroblasts in wound repair process (granulation tissue). ${ }^{*}$ original, ${ }^{* *}$ proposal by the present author.

invasion and metastasis. Our proposal suggests that we need another standpoint in the study of the expression of various matrix-degrading enzymes in cancer tissue, adopting a comparative study between cancer and wound repair process. Our recent paper emphasized the paucity of stromal cells positive for MMP-9 and urokinase-type plasminogen activator receptor (uPAR) in diffuse-type gastric cancer (Migita et al. 1999). Increased deposition of collagen fibers and diffuse growth of dissociated cancer cells characterize this type of cancer. If MMP-9 or uPAR promote cancer invasion, cells positive for these are expected to be higher in diffuse-type than in other type (intestinal type) of gastric cancer. The results were opposite. We considered the results from the viewpoint matrix turnover (remodeling). Remodeling process is regarded to be more quiescent in diffusetype.

The distinction of angiogenesis: Nutrient type and immune/inflammatory type

Angiogenesis is prerequisite for the development of solid tumor as nutrient vessels. In inflammatory lesions, another angiogenesis occurs as inflammatory angiogenesis, where one finds venules which express the cell adhesion molecules. In inflammatory disease or in immune responses, these adhesion molecules (ICAM1, VCAM-1 and E-selectins) are required for the transmigration of leukocytes from blood stream to the tissue (Granger 1997; McGill et al. 1998; Salmi et al. 1998). These vessels are regarded as venules activated by inflammation (Nakamura et al. 1993). In accordance with this concept, the area of invasive margin of colorectal cancer is a site of active inflammation infiltrated with neutrophils, macrophages, and lymphocytes together with "activated venules" (Suzuki et al. 1995). Blood 
vessels were clearly detected both in the stroma of cancer and along the invasive margin by laminin staining which labels the basal lamina of endothelial cells and pericytes/smooth muscle cells. E- and P-selectins are positive in venules along the invasive margin. Since ICAM- 1 is expressed by many inflammatory cells and stromal fibroblasts, ICAM-1 did not specify blood vessels. Invasive margin is an area infiltrated by above-mentioned cells, therefore, we speculated that venules positive for the cell adhesion molecules are also venules activated to facilitate the transmigration of leukocytes. A concept essentially the same as ours has been proposed by experimental studies on tumor immunity (Ogawa et al. 1997, 1998). Contrasted to this, stromal blood vessels within cancer tissue lacked the expression of selectins. This can be interpreted that blood vessels in cancer stroma may be suppressed from the immunologic standpoint. This study stressed the differences of the two kinds of blood vessels in cancer tissue for the first time. The patholophysiologic significance will be described in the next section.

\section{Immune reactions in cancer tissue}

First we analyzed Epstein-Barr virus (EBV)-associated gastric cancer. In Japan, approximately $7 \%$ of gastric cancer is associated with the latent infection of EBV (Tokunaga et al. 1993). The typical form of this type of cancer is medullary carcinoma with lymphoid stroma. Most of this type is EBV-associated cancer. Our study revealed that EBV-associated cancer is infiltrated with CD8 ${ }^{+}$ T-cells within cancer cell nests bearing a high proliferation activity (Saiki et al. 1996). We further confirmed the presence of perforin granules in these cells. These data indicated the presence of reactions of activated cytotoxic T-cells in $\mathrm{EBV}$-associated gastric cancer, suggesting that $\mathrm{EBV}$-associated gastric cancer is an immunogenic tumor.

Next, we analyzed possible antigen-presenting cells in cancer tissue by immunostaining of B7-1 (CD80) and B7-2 (CD86) costimulatory molecules. The costimulatory molecules are required to convey the proliferation signals to T-cells which recognize antigens expressed on the antigen presenting cells (Croft and Dubey 1997). First, we have found that epithelioid granuloma cells in Crohn's disease express B7.1 and B7.2 with close cell-to-cell contact to both CD4 and CD8 ${ }^{+}$ T-cells (Hara et al. 1997). We then analyzed colon cancer tissue, since we know that macrophages are abundantly distributed along the invasive margin of colorectal cancer, which are positive for the intercellular adhesion molecule-1 (ICAM-1) (Mizoi et al. 1995). These cells are also positive for B7.2 costimulatory molecule (Ohtani et al. 1997). T-cells, which can receive antigenic stimuli, are co-localized in the same area. Double immunohistochemistry revealed close cell-to-cell contact between $\mathrm{B} 7-2^{+}$cells and T-cells. These observations suggested an occurrence of immune reaction there. Their location suggests that tumor antigens would be included in the antigens recognized. These macrophages were more abundant in colorectal cancer without liver metastasis than that with liver metastasis (Takeha 
et al. 1997). This suggested that these macrophages are inhibitory for the distant metastasis via hematogenous route.

Dendritic cells (DCs) are key cells that modulate the immune reactions (Banchereau and Steinman 1998). Immature DCs bear an ability of phagocytosis of apoptotic cells equipped with lysosomal enzymes (antigen capture and processing) (Albert et al. 1998). Upon maturation, DCs express MHC class II and B7 molecules on the cell surface. This poses complexity in the nomenclature of B7.2+ "macrophages" in our study. Cells observed by us express MHC class II, B7.2, ICAM-1, and CD68, but lacks CD83 (unpublished data), a marker of mature DCs. This indicates that $\mathrm{B} 7.2^{+}, \mathrm{CD} 68^{+}$cells we observed are not mature DCs, probably activated macrophages. More detailed studies will clarify the relationship between the two cells.

The staining of B7 is only possible with frozen sections. Therefore, we need other marker to perform a clinicopathologic analysis. As a possible effetor cell, we selected CD8 ${ }^{+}$T-cells. Based on the observations in EBV-associated gastric cancer, we classified $\mathrm{CD}^{+}$T-cells by their locations into three; those within cancer cell nests (intraepithelial), those in the stroma and those along the invasive margin. The number is most abundant in those along the invasive margin. $\mathrm{CD}^{+}$T-cells were scored into IV groups after either quantification or semiquantification, and relations with the patients' survival were analyzed. A study of 131 patients with colorectal cancer revealed that the CD8 ${ }^{+} \mathrm{T}$-cells within cancer cell nests had the most significant impact on the patients' survival; the more CD8, the better the patients' survival by univariate analysis. The multivariate analysis confirmed that the impact of this factor is similar to that of the Dukes' staging. Double staining of CD8 and the proliferation-associated antigen, $\mathrm{Ki}-67$ revealed that the labeling index of $\mathrm{CD}^{+} \mathrm{T}$-cells within cancer cell nests is $6 \%$, higher than those along the invasive margin $(2 \%)$. This suggests that these T-cells are activated. Granzyme B immunohistochemisty revealed that there are sparsely granulated cells within cancer cell nests, suggesting the presence of activated cytotoxic cells. These data indicate the presence of a certain anti-tumor immunity, functioning to diminish the aggressiveness of cancer (Naito et al. 1998) (Fig. 2).

Poorly differentiated colorectal adenocarcinoma, a rare histologic type in colorectal cancer, has a higher positivity rate for microsatellite instability (MSI) than in usual colorectal cancer (Ruschoff et al. 1997). Collaboration with Dr. A. Horii's group (Division of Molecular Pathology, Tohoku University School Medicine) showed that CD8 ${ }^{+} \mathrm{T}$-cells within cancer cell nests are significantly larger in number in $\mathrm{MSI}^{+}$group in poorly differentiated colorectal cancer (Wakatsuki et al., manuscript in preparation). MSI can induce frame shift mutations, which can produce antigens to be targeted by immune cells. We have found a certain mechanism linking genetic alterations and immune reactions.

Our ongoing studies are revealing that the number of $\mathrm{CD}^{+} \mathrm{T}$ cells within 


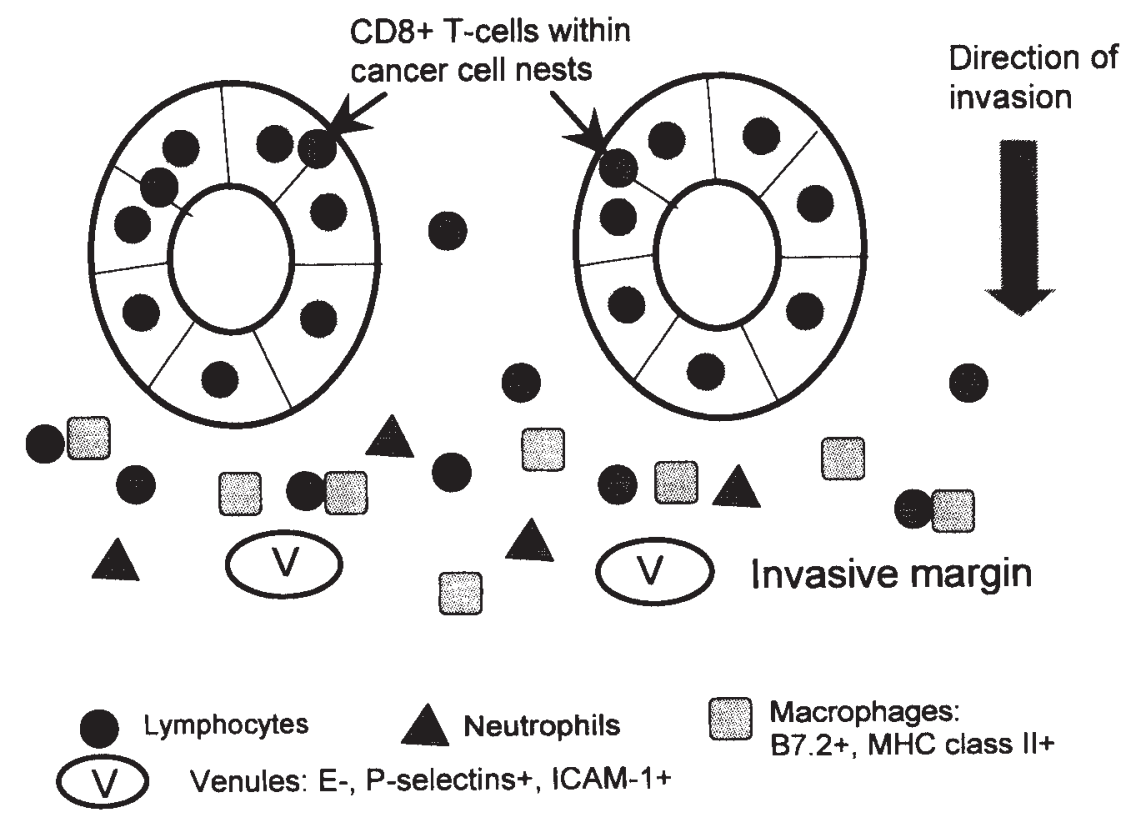

Fig. 2. Cancer invasion and host immune/inflammatory reactions (colorectal cancer).

Venules along the invasive margin express the cell adhesion molecules. Macrophages, neutrophils and lymphocytes (mostly T-cells) are distributed along the invasive margin. $\mathrm{B} 7.2^{+}$macrophages and T-cells are frequently in a close cell-to-cell contact. Arrows indicate $\mathrm{CD}^{+}{ }^{+} \mathrm{T}$-cells within cancer cell nests. The number of these lymphocytes is related to a better survival of patients.

cancer cell nests seems to be higher in poorly differentiated histologic types than in well-differentiated types in lung and kidney cancers. This may be related to one of basic features of cancerization, which awaits further analysis.

\section{ConCLUSION}

The biologic behavior of cancer is not only determined by cancer cell factors but also by various host reactions. Host reactions modulate the growth and spread of cancer in various manners. We need comprehensive analysis.

\section{Acknowledgments}

I am grateful to the following collaborators who continued to investigate with me and encourage me: Takayuki Mizoi, Yukimasa Suzuki, Takashi Migita, Kazuya Saito, Shiro Nakamura, Kouhei Fukushima, Satoshi Takeha, Shinji Kumagai, Yoshitaka Naito, Yuriko Saiki, Eiichi Sato (our laboratory); Masayuki Yamamoto, Kazuhiko Igarashi, Tadashi Nagai, Hozumi Motohashi (Dept. of Biochemistry, Tohoku University School of Medicine); Kennichi Shiiba (Dept. of Surgery I, Tohoku University School of Medicine); Akio Ohuchi (Tohoku Rosai Hospital); Noriaki Ohuchi, Naohiro Ariga (Dept. of Surgery II, Tohoku University School of Medicine); Atsushi Kuroiwa (Nagoya); Motoharu Seiki (Tokyo); Masaaki Miyazawa (Osaka), Keld Dan $\phi$ (Copenhagen); Timo Sorsa (Helsinki); Wen-Tien Chen (New York) and Hiroshi Nagura. 


\section{References}

1) Albert, M.L., Sauter, B. \& Bhardwaj, N. (1998) Dendritic cells acquire antigen from apoptotic cells and induce class I-restricted CTLs. Nature, 392, 86-89.

2) Banchereau, J. \& Steinman, R.M. (1998) Dendritic cells and the control of immunity. Nature, 392, 245-252.

3) Croft, M. \& Dubey, C. (1997) Accessory molecule and costimulation requirements for CD4 T cell response. Crit. Rev. Immunol., 17, 89-118.

4) Dvorak, H.F. (1986) Tumors: Wounds that do not heal. Similarities between tumor stroma generation and wound healing. N. Engl. J. Med., 315, 1650-1659.

5) Goldstein, L.A., Ghersi, G., Pineiro-Sanchez, M.L., Salamone. M,, Yeh, Y., Flessate, D. \& Chen, W-T. (1997) Molecular cloning of seprase: A serine integral membrane proteiase from human melanoma. Biochim. Biophys. Acta, 1361, 11-19.

6) Granger, D.N. (1997) Cell adhesion and migration. II. Leukocyte-endothelial cell adhesion in the digestive system. Am. J. Physiol., 273, 982-986.

7) Hara, J., Ohtani, H., Matsumoto, T., Nakamura, S., Kitano, A., Arakawa, T., Nagura, H. \& Kobayashi, K. (1997) Expression of costimulatory molecules B7-1 and B7-2 in macrophages and granulomas of Crohn's disease: Demonstration of cell-to-cell contact to T-lymphocytes. Lab. Invest., 77, 175-184.

8) Heppner, K.J., Matrisian, L.M., Jensen, R.A. \& Rodgers, W.H. (1996) Expression of most matrix metalloproteinase family members in breast cancer represents a tumorinduced host response. Am. J. Pathol., 149, 273-282.

9) McGill, S.N., Ahmed, N.A. \& Christou, N.V. (1998) Endothelial cells: Role in infection and inflammation. World J. Surg., 22, 171-178.

10) Migita, T., Sato, E., Saito, K., Mizoi, T., Shiiba, K., Matsuno, S., Nagura, H. \& Ohtani, H. (1999) Differing expression of MMPs-l and 9, and urokinase receptor between diffuse- and intestinal-type gastric carcinoma. Int. J. Cancer, 84, 74-79.

11) Mizoi, T., Ohtani, H., Miyazono, K., Miyazawa, M., Matsuno, S. \& Nagura, H. (1993) Immunoelectron microscopic localization of transforming growth factor $\beta 1$ and latent transforming growth factor $\beta 1$ binding protein in human cancer cells and stromal cells. Cancer Res., 53, 1-8.

12) Mizoi, T., Ohtani, H., Suzuki, Y., Shiiba, K., Matsuno, S. \& Nagura, H. (1995) Intercellular adhesion molecule-1 expression by macrophages in human gastrointestinal carcinoma: Possible roles as host immune/inflammatory reaction. Pathol. Int., 45, 565-572.

13) Naito, Y., Saito, K., Shiiba, K., Ohuchi, A., Saigenji, K., Nagura, H. \& Ohtani, H. (1998) CD8 + T-cells infiltrated within cancer cell nests as a prognostic factor in human colorectal cancer. Cancer Res., 58, 3491-3494.

14) Nakamura, S., Ohtani, H., Watanabe, Y., Fukushima, K., Matsumoto, T., Kitano, A., Kobayashi, K. \& Nagura, H. (1993) In situ expression of the cell adhesion molecules in inflammatory bowel disease: Evidence of immunologic activation of vascular endothelial cells. Lab. Invest., 69, 77-85.

15) Ogawa, M., Tsutsui, T., Zou, J.P., Mu, J., Wijesuriya, R., Yu, W.G., Herrmann, S., Kubo, T., Fujiwara, H. \& Hamaoka, T. (1997) Enhanced induction of very late antigen 4/lymphocyte function-associated antigen 1-dependent T-cell migration to tumor sites following administration of interleukin 12. Cancer Res., 57, 2216-2222.

16) Ogawa, M., Yu, W.G., Umehara, K., Iwasaki, M., Wijesuriya, R., Tsujimura, T., Kubo, T., Fujiwara, H. \& Hamaoka, T. (1998) Multiple roles of interferon-gamma in the mediation of interleukin 12-induced tumor regression. Cancer Res., 58, 2426-2432.

17) Ohtani, H. \& Sasano, N. (1983) Stromal cell changes in human colorectal adenomas and carcinomas: An ultrastructural study of fibroblasts, myofibroblasts and smooth muscle cells. Virchows Arch., 401, 209-222. 
18) Ohtani, H., Kuroiwa, A., Obinata, M., Ooshima, A. \& Nagura, H. (1992) Identification of type I collagen producing cells in human gastrointestinal carcinoma by non-radioactive in situ hybridization and immunoelectron microscopy. J. Histochem. Cytochem., 40, 1139-1146.

19) Ohtani, H., Nagai, T. \& Nagura, H. (1995) Similarities of in situ mRNA expression between gelatinase A (MMP-2) and type I procollagen in human gastrointestinal carcinoma: Comparison with granulation tissue reaction. Jpn. J. Cancer Res., 86, 833-839.

20) Ohtani, H., Naito, Y., Saito, K. \& Nagura, H. (1997) Expression of costimulatory molecules B7-1 and B7-2 by macrophages along invasive margin of colon cancer: A possible anti-tumor immunity? Lab. Invest., 77, 231-241.

21) Ohtani, H. (1998) Stromal reaction in cancer tissue: Pathophysiologic significance of expression of matrix-degrading enzymes in relation to matrix turnover and immune/inflammatory reactions. Pathol. Int., 48, 1-9.

22) Pineiro-Sanchez, M.L., Goldstein, L.A., Dodt, J., Howard, L., Yeh, Y. \& Chen, W-T. (1997) Identification of the $170-\mathrm{kDa}$ melanoma membrane-bound gelatinase (seprase) as a serine integral membrane protease. J. Biol. Chem., 272, 7595-7601.

23) Poulsom, R., Pignatelli, M., Stetler-Stevenson, W.G., Liotta, L.A., Wright, P.A., Jeffery, R.E., Longcroft, J.M., Rogers, L. \& Stamp, G.W.H. (1992). Stromal expression of $72-\mathrm{kDa}$ type-IV-collagenase (MMP-2) and TIMP-2 mRNAs in colorectal neoplasia. Am. J. Pathol., 41, 389-396.

24) Pyke, C., Ralfkiaer, E., Huhtala, P., Hurskainen, T., Dano, K. \& Tryggvason, K. (1992) Localization of messenger RNA for 72-kDa and 92-kDa-type IV collagenases in human skin cancers by in situ hybridization. Cancer Res., 52, 1336-1341.

25) Ruschoff, J., Dietmaier, W., Luttges, J., Seitz, G., Bocker, T., Zirngibl, H., Schlegel, J., Schackert, H.K., Jauch, K.W. \& Hofstaedter, F. (1997) Poorly differentiated colonic adenocarcinoma, medullary type: Clinical, phenotypic, and molecular characteristics. Am. J. Pathol., 150, 1815-1825.

26) Saiki, Y., Ohtani, H., Naito, Y., Miyazawa, M. \& Nagura, H. (1996) Immunophenotypical characterization of Ebstein-Barr virus-associated gastric carcinoma: Massive infiltration by proliferating CD8 + T-lymphocytes. Lab. Invest., 75, 67-76.

27) Sakakibara, K., Suzuki, T., Motoyama, T., Watanabe, H. \& Nagai, Y. (1982) Biosynthesis of an interstitial type of collagen by cloned human gastric carcinoma cells. Cancer Res., 42, 2019-2027.

28) Salmi, M., Adams, D. \& Jalkanen, S. (1998) Cell adhesion and migration. IV. Lymphocyte trafficking in the intestine and liver. Am. J. Physiol., 274, 1-6.

29) Scanlan, M.J., Raj, B.K., Calvo, B., Garin-Chesa, P., Sanz-Moncasi, M.P., Healey, J.H., Old, L.J. \& Rettig, W.J. (1994) Molecular cloning of fibroblast activation protein alpha, a member of the serine protease family selectively expressed in stromal fibroblasts of epithelial cancers. Proc. Natl. Acad. Sci. USA, 91, 5657-5661.

30) Shull, M.M., Ormsby, I., Kier, A.B., Pawlowski, S., Diebold, R.J., Yin, M., Allen, R., Sidman, C., Proetzel, G. \& Calvin, D. (1992) Targeted disruption of the mouse transforming growth factor-beta 1 gene results in multifocal inflammatory disease. Nature, 359, 693-699.

31) Suzuki, Y., Ohtani, H., Mizoi, T., Takeha, S., Shiiba, K., Matsuno, S. \& Nagura, H. (1995) Cell adhesion molecule expression by vascular endothelial cells as an immune/inflammatory reaction in human colon carcinoma. Jpn. J. Cancer Res., 86, $585-593$.

32) Takeha, S., Fujiyama, Y., Bamba, T., Sorsa, T., Nagura, H. \& Ohtani, H. (1997) Stromal expression of MMP-9 and urokinase receptor is inversely associated with liver metastasis and with infiltrating growth in human colorectal cancer: A novel approach from immune/inflammatory aspect. Jpn. J. Cancer Res., 88, 72-81.

33) Tokunaga, M., Land, C.E., Uemura, Y., Tokudome, T., Tanaka, S. \& Sato, E. (1993) 
Epstein-Barr virus in gastric carcinoma. Am. J. Pathol., 143,: 1250-1254. 